\title{
HIPOXIA APÓS TRAUMATISMOS CRÂNIO-ENCEFÁLICOS GRAVES
}

\author{
R. A. Frowein
}

\author{
A. KARIMI \\ K. H. EULER
}

Apesar de todos os modernos métodos de tratamento, a mortalidade após traumatismos crânio-encefálicos graves ainda é da ordem de 50 a $70 \%$ dos casos (Frowein 11). Para modificar estes resultados as possibilidades clinicas parecem ser muito limitadas porém ainda não esgotadas. A hipoxia é um dos fatôres mais importantes a considerar.

$\mathrm{Na}$ fase aguda de traumatismos encefálicos há diminuição do afluxo de $\mathrm{O}_{2}$ ao cérebro, consecutiva a distúrbios respiratôrios e circulatórios. Daí resulta, inicialmente, uma alteração na atividade dos neurônios e, ulteriormente, tôda uma cadeia de distúrbios funcionais do tecido encefálico (Opitz e Schneider ${ }^{25}$, Schneider ${ }^{30}$, Gänshirt ${ }^{16}$, Frowein ${ }^{9}$ ).

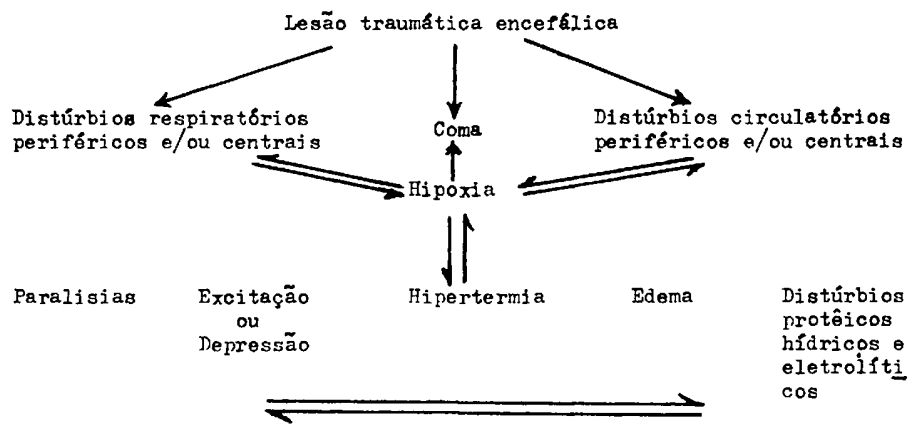

Quadro 1 - Hipoxia como conseqüência de traumatismos crânioencefálicos e como causa de distúrbios da regulação do metabolismo (apud Strughold ${ }^{35}$ ).

A terapêutica dos distúrbios circulatórios após severos traumatismos cerebrais ainda não está inteiramente solucionada apesar do emprêgo precoce de infusões e de ganglioplégicos (Frowein ${ }^{14}$, Schürmann ${ }^{32}$ ).

Trabalho feito na Clínica Neurocirúrgica da Universidade de Colônia e no Instituto Max Planck para Pesquisas Encefálicas, Secção de Tumores e Patologia Experimental (Prof. Dr. W. Tönnis), publicado no Zentralblatt für Neurochirurgie 23(1) 1962, traduzido do alemāo pelo Dr. Guilherme Cabral, Assistente de Clínica Neurológica na Faculdade de Medicina da Universidade de Minas Gerais. 
Melhoras são proporcionadas pelas medidas que atuam corrigindo os disdistúrbios respiratórios periféricos e também pela desobstrução das vias respiratórias. Contudo, ainda são insuficientes os estudos sôbre os distúrbios respiratórios centrais que ocorrem na fase aguda dos traumatismos crânioencefálicos. É muito difundida a opinião de que nos traumatismos graves há sempre uma respiração insuficiente; aparentemente esta hipoventilação ocorre apenas nos casos em que há hipotonia muscular generalizada e nos quais o espirograma mostra que são pequenas as incursões respiratórias e que o volume-minuto é insuficiente. Entretanto, em muitos traumatizados, especialmente nos casos com intensa excitação motora e aumento do tono muscular (convulsões e contraturas) também ocorre hipoventilação (Frowein ${ }^{15}$ ), idênticamente ao que sucede em outras lesões cerebrais agudas como, por exemplo, nas hemorragias cerebrais (Heyman e col. ${ }^{19}$, Plum e Swanson ${ }^{27}$, Brown e Plum ').

Resta verificar se em tais distúrbios da respiração o suprimento de oxigênio do encéfalo é normal, ou não. Para isto pode-se medir a quantidade de $\mathrm{O}_{2}$ do sangue e a saturação arterial.

A figura 2 mostra os valôres de 118 medições do teor percentual de $O_{z}$ em diferentes dias após traumatismos crânio-encefálicos graves. Em alguns casos encontraram-se valôres acentuadamente reduzidos (abaixo de $85 \%$ ) devido a sérios distúrbios respiratórios motivados por obstrução das vias aéreas periféricas e consecutivo edema pulmonar e broncopneumonia. Mediante administração de $\mathrm{O}_{2}$ e, às vêzes, após traqueostomia, os valôres de saturação arterial melhoraram nìtidamente, como também tinha sido demonstrado por Schnedorf e col. em $1940^{28}$.

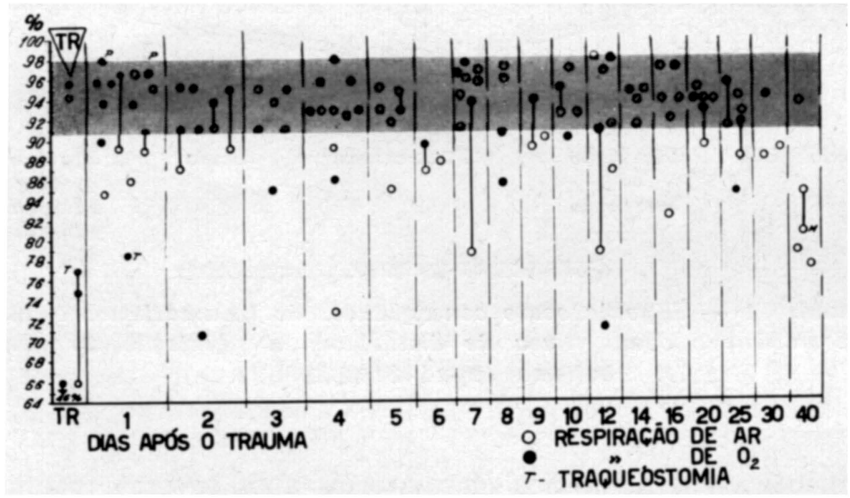

Fig. 1 - A saturação percentual de $O$. no sangue arterial $\dot{e}$ habitualmente normal $(90$ a $98 \%)$ nos traumatismos encefalicos, depois de ter sido feita a desobstruç̃o das vias aéreas e administrado oxigênio.

$\mathrm{Na}$ maioria dos casos em fase aguda a saturação de oxigênio permanece entre os niveis percentuais de 90 a 99\% (Bartels e col. ${ }^{3}$, Anthony e Venrath ${ }^{1}$ ), o que não nos garante que haja pressão normal de oxigênio no te- 
cido cerebral. Por isso as pesquisas subseqüentes visam confrontar a saturação arterial de oxigênio com o teor do afluxo sangüíneo ao encéfalo, com as alterações do $\mathrm{pH}$ arterial e da tensão de $\mathrm{CO}_{2}$, com as modificações do teor hemoglobínico do sangue.

O afluxo sangüíneo ao tecido cerebral depende da relação entre a pressão arterial média e a pressão intracraniana (Kety e col. ${ }^{21}$, Noell e Schneider ${ }^{24}$, Opitz e Schneider ${ }^{25}$, Bernsmeier e Siemons ${ }^{4}$, Gänshirt e Tönnis ${ }^{17}$, Gänshirt ${ }^{16}$ ). A circulação cerebral é diminuída pelo aumento da pressão intracraniana como o demonstra o retardamento circulatório verificado durante a prática de angiografias seriadas (Tönnis e Schiefer ${ }^{36}$, Frowein ${ }^{10}$ ). Mas mesmo que não ocorram modificações do afluxo sangüíneo é de se temer que surjam sérias alterações no suprimento de oxigênio ao cérebro graças a modificações da composição do sangue e do equilíbrio ácido-básico.

Hemogramas feitos em 34 traumatizados crânio-encefálicos na fase aguda mostraram, às vêzes, a existência de anemia mais intensa do que se poderia prever. Diminuição do número de eritrócitos abaixo de 3 milhões/

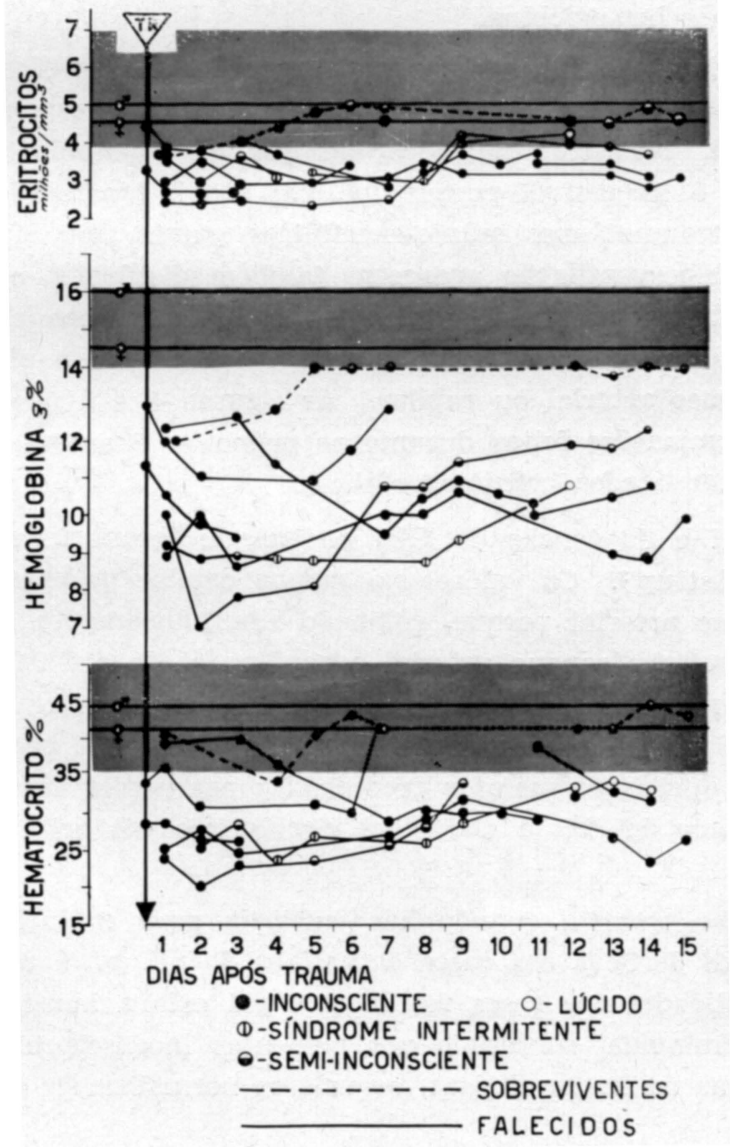

Fig. 2 - Modificaçóes no número de hemácias, do teor de hemoglobina $e$ do hematócrito após traumatismos crânio-encefálicos graves. 
$\mathrm{mm}^{3}$ e queda da hemoglobina abaixo de $10 \mathrm{~g} \%$ ocorrem sobretudo em feridos graves com prolongado estado de inconsciência e evolução fatal (fig. 3). A diminuição concomitante do hematócrito demonstra a diluição do sangue que já havia sido verificada por Pampus ${ }^{26}$.

Pacientes traumatizados com evolução não complicada apresentaram valôres normais ou quase normais da série vermelha; valôres acima do normal foram apenas observados em casos isolados, sendo a hemoconcentração fácil de combater com o uso de infusões salinas.

Nossas observações de que as anemias são mais freqüentes após os traumatismos cranianos graves concordam com os achados de Smolik e col. ${ }^{33,34}$, obtidos também em casos de traumatismos crânio-encefálicos, e com os de Gelin ${ }^{18}$, obtidos em outros traumatismos graves e em casos de queimaduras extensas.

Deve-se assinalar que quando ocorre diminuição de hemoglobina para $10 \%$, mesmo que a saturação arterial de oxigênio seja daa ordem de $95 \%$, o conteúdo real de oxigênio corresponde a apenas $63 \%$ de um sangue com o conteúdo normal de $15 \%$ de hemoglobina.

Estas alterações não seriam tão importantes se a redução do conteúdo de oxigênio do sangue pudesse ser equilibrado por um aumento correspondente da circulação cerebral. Nos traumatizados em que há hipotonia e hipoventilação tal equilíbrio é impossivel porque há, em geral, hipotensão arterial concomitante. Nos traumatismos crânio-encefálicos graves com aumento do tono muscular e hiperventilação aparecem também distúrbios no equilíbrio ácido-básico que determinam diminuição do afluxo sangüíneo ao encéfalo; estes distúrbios podem ser encontrados quando se determina o $\mathrm{pH}$ e a tensão do $\mathrm{CO}_{2}$ nos sangues arterial ou capilar. As figuras 4 e 5 mostram os valôres de 276 determinações feitas durante as primeiras 3 semanas após o acidente, em 69 traumatizados crânio-encefálicos.

As determinaçōes do $\mathrm{pH}$ e da tensão do $\mathrm{CO}_{2}$ no sangue foram feitas com o micro-analisador de Astrup ${ }^{2}$. Os valôres no sangue capilar puderam ser justapostos aos do sangue arterial porque, colhendo simultâneamente, a diferença que encontramos entre o sangue arterial e capilar foi de até 0,02 unidades de $\mathrm{pH}$ (o $\mathrm{pH}$ normal pode ter variação de até 0,08 unidades). A diferença arterial/capilar em pessoas normais, segundo Maas e Heijst ${ }^{22}$, pode ser de 0,0023 até 0,0075 ; as diferenças maiores encontradas nos nossos casos puderam ser consideradas como devidas a distúrbios circulatórios de origem central.

Nos dias subseqüentes ao trauma, quando há tendência para evolução satisfatória (fig. 3), em cêrca de $50 \%$ dos casos os valôres do pH permaneceram nos limites da normalidade; nos casos restantes o $\mathrm{pH}$ estava aumentado e a tensão de $\mathrm{CO}_{2}$ diminuída, sobretudo nos pacientes inconscientes; entretanto, coincidindo com as melhoras clínicas, os valôres normalizaram-se. 


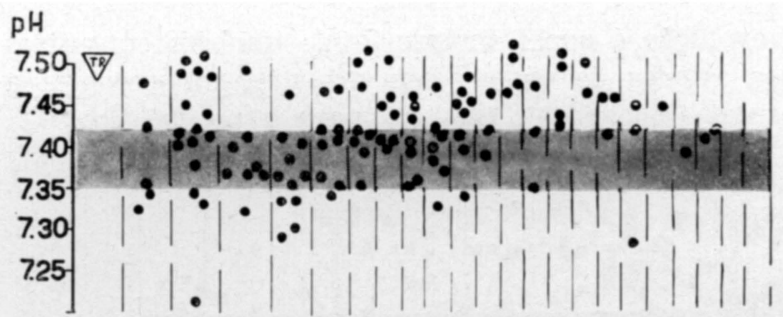

Sland $\mathrm{HCO}_{3}$
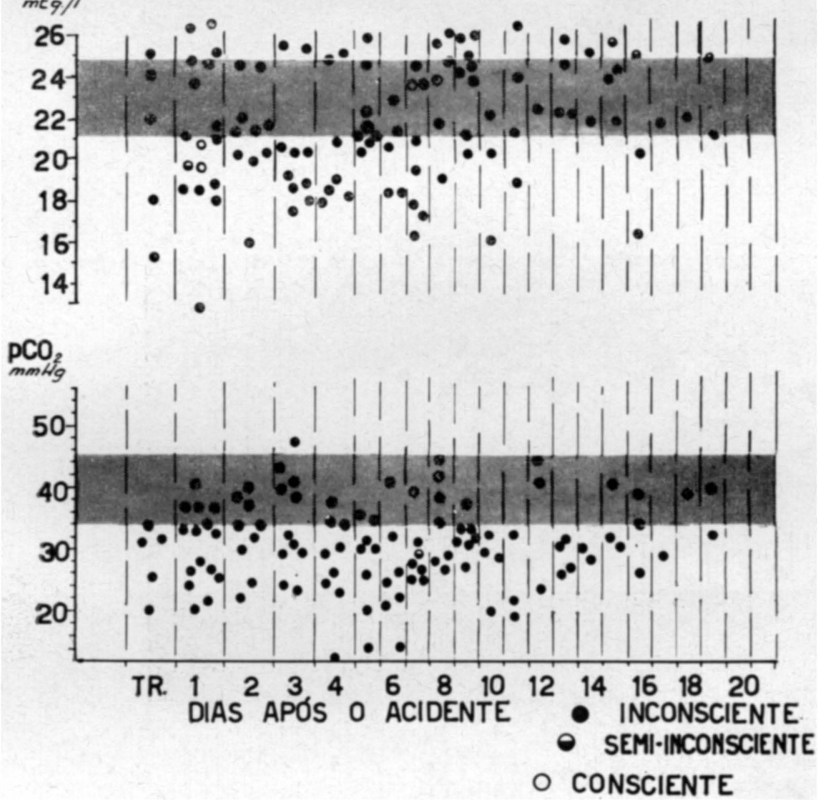

Fig. 3 - Distúrbios do equilíbrio ácido-básico no sangue arterial de pacientes com graves traumatismos crânioencefálicos. Na evolução ocorre freqüentemente alcalose respiratória e mista que regride se houver tendência à melhora. Todos êstes pacientes sobreviveram.

Em traumatizados que faleceram ulteriormente (fig. 4) verificou-se que quando havia persistência do estado de inconsciência não ocorria esta normalização; os valôres do $\mathrm{pH}$ permaneciam muito aumentados e os valôres da tensão do $\mathrm{CO}_{2}$ no sangue permaneciam diminuídos.

A medição da reserva alcalina mostrou que ocorre alcalose respiratória e mista nos casos em que há aumento da respiração conseqüente à excitabilidade exagerada dos centros respiratórios (Frowein 12, 1.3).

Em alguns casos de traumatismos graves os valôres do $\mathrm{pH}$ durante os primeiros dias após o trauma ou na fase final apresentaram-se nittidamente diminuídos, principalmente como conseqüência de acidose metabólica descompensada ou mista. Ao contrário das nossas observações, Cook e col. ${ }^{8}$ encontraram, em 16 traumatizados crânio-encefálicos graves, uma acidose respiratória e mista não apenas nas fases iniciais como também na evolução ulterior. As observações porém concordam no fato de que nos traumatismos 
encefálicos com acidose a evolução é quase sempre fatal; também em outros traumatismos graves e nos estados de choque sem traumatismo cerebral a acidose é achado fundamental (Wiggers ${ }^{37}$, Bland ${ }^{6}$ ) para o prognóstico.

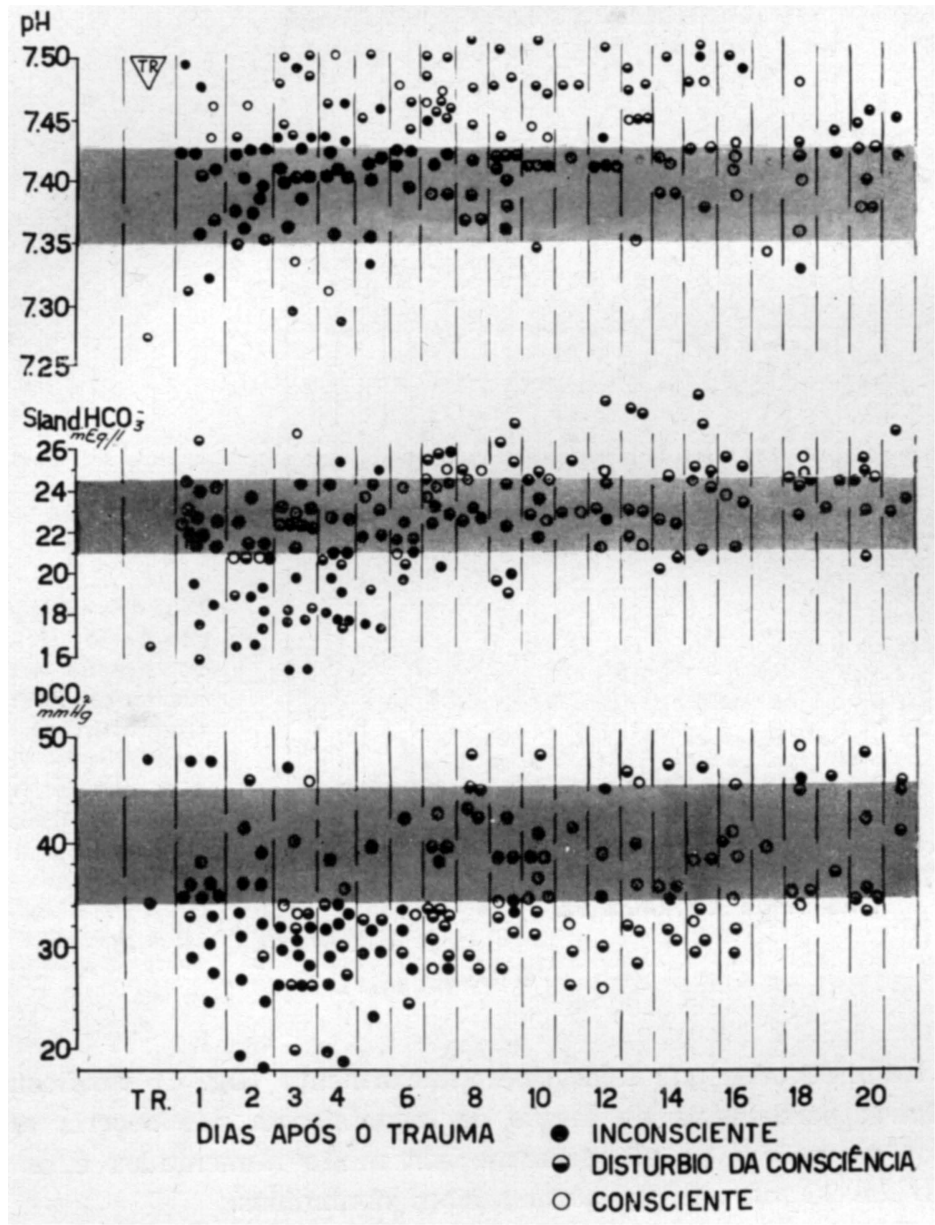

Fig. 4 - Distúrbios do equilibrio ácido-básico no sangue arterial de pacientes com graves traumatismos crânio-encefálicos. Ao contrário do que mostra a figura 3, na maioria dos casos que evoluem para o êxito letal, não há diminuição do pH e aumento do $\mathrm{PCO}$, no sangue arterial.

Os desvios do $\mathrm{pH}$ e do $\mathrm{pCO}_{2}$ na evolução dos traumatismos crânio-encefálicos graves têm importante e duplo significado clínico: de um lado mostram que as medidas terapêuticas atuais, até mesmo a desconexão neurovegetativa continua com fenotiazina e outros neuroplégicos, ainda não possibilitam inteira normalização das funções vitais, sobretudo da respira- 
são; em segundo lugar, a redução da tensão do $\mathrm{CO}_{2}$ arterial determina distúrbics da circulação periférica e, sobretudo, diminuição do afluxo sangüíneo ao cérebro (Kety e col. ${ }^{21}$, Frowein ${ }^{13}$ ). A hiperventilação provoca também uma agregação eritrocitária nos capilares determinando estase (Nielsen ${ }^{23}$ ) e hipoxia dos tecidos (Schneider ${ }^{30}$, Bischof ${ }^{5}$ ). Daí decorre que, mesmo nos traumatizados que clìnicamente apresentam uma respiração satisfatória para a saturação arterial de oxigênio, o suprimento de oxigênio do encéfalo pode estar comprometido. Deve-se assinalar que nos traumatizados graves pode-se regular adequadamente a tensão de $\mathrm{O}_{2}$ do sangue arterial, mas, devido à anemia ou à deficiência da circulação cerebral, nem sempre é possivel um aporte adequado de $\mathrm{O}_{2}$ para o cérebro.

A tensão do $\mathrm{O}_{2}$ no sangue venoso é de maior importância para a oxigenação celular. A tensão normal de oxigênio no sangue venoso cerebral é de 34-36 mm Hg e quando esta tensão é menor, deixa de ser normal a tensão média do oxigênio no tecido encefálico. Até uma tensão venosa de $28 \mathrm{~mm} \mathrm{Hg}$ ainda existe, segundo Opitz e Schneider ${ }^{25}$, uma zona indiferente; valôres mais baixos determinam alterações reversiveis no sistema nervoso central, como sejam, por exemplo, alteraçōes no EEG. Quando a tensão do $\mathrm{O}_{2}$ baixa para menos de $19 \mathrm{~mm}$ de $\mathrm{Hg}$ começa a zona critica com distúrbios da consciência (Opitz e Schneider ${ }^{25}$, Gänshirt ${ }^{16}$, Hirsch e col. ${ }^{20}$ ).

Pudemos fazer até agora determinações da tensão do oxigênio no sangue venoso do encéfalo em 4 pacientes inconscientes, sendo três em conseqüência de graves traumatismos crânio-encefálicos e um após intervenção cirúrgica (fig. 5) : em 3 casos o valor da tensão do $\mathrm{O}_{2}$ venoso cerebral estava situado pouco abaixo dos limites da normalidade, no limite inferior do setor indife-
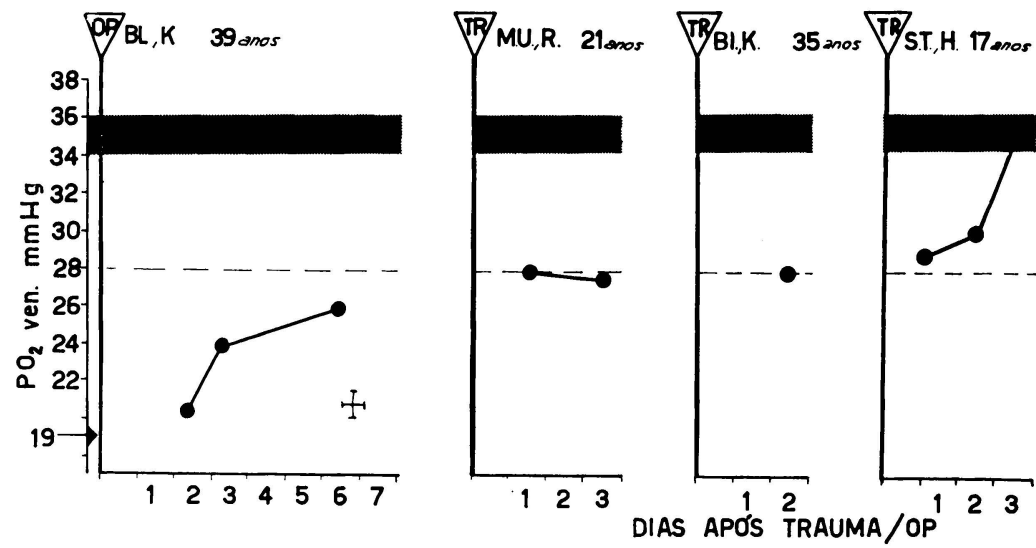

Fig. 5 - Tensão de $\mathrm{O}_{2}$ no sangue venoso do encéfalo em 4 pacientes em estado de inconsciência. Apesar de estarem as vias aéreas desobstruídas $e$ mesmo na vigência da administração de $O_{z}$, as tensões estõo abaixo do normal (34 a $36 \mathrm{~mm} \mathrm{Hg})$ : nos 3 casos em que a evolucão foi favorável a tensão era de $28 \mathrm{~mm} \mathrm{Hg}$ aproximadamente; em um caso de evolução fatal a tensão de $\mathrm{O}_{2}$ no sangue venoso era de $19 \mathrm{~mm} \mathrm{Hg}$. 
rente, e êstes 3 pacientes sobreviveram; no caso restante, de evolução fatal, a tensão do $\mathrm{O}_{2}$ no sangue venoso encefálico estava acentuadamente reduzida, transitòriamente até mesmo nos limites da zona crítica, embora tudo tenha sido feito para a desobstrução das vias aéreas e manutenção de uma circulação cerebral satisfatória (traqueostomia, aspiração de secreções, administração de $\mathrm{O}_{2}$, infusōes, transfusōes de sangue).

\section{RESUMO}

Após traumatismos crânio-encefálicos graves ocorre hipoxia cerebral, não apenas nas primeiras horas mas também durante vários dias da fase aguda. A saturação arterial de $\mathrm{O}_{2}$ é muitas vêzes normal, mas, com mais freqüência do que se supõe, existe anemia; como conseqüência do aumento da pressão intracraniana, da hipotensão arterial ou da alcalose respiratória, diminui o afluxo sangüíneo ao encéfalo, baixando a tensão do $\mathrm{O}_{2}$ celular, o que é demonstrado pela diminuição da tensão de $\mathrm{O}_{2}$ no sangue venoso provindo do encéfalo. Por isso, no tratamento da fase aguda pós-traumática deve-se, ao lado da normalização da pressão intracraniana, manter a pressão arterial, evitar a hiperventilação pulmonar mediante a administração de neuroplégicos e impedir a anemia mediante transfusões de sangue.

\section{SUMMARY}

Hypoxia after severe skull-brain injuries.

After severe skull-brain injuries hypoxia occurs not only during the first hours but even for several days during the acute stage. Though the arterial oxygen saturation is mostly normal, cerebral anemia is present more often than it could be expected: owing to the rise of intracranial pressure, the fall of blood pressure and/or respiratory alkaiosis, the cerebral circulation is reduced and the oxygen pressure for the brain cells consequently falls; this was proved by measuring the $\mathrm{O}_{2}$ tension of the venous blood of the brain. Therefore after severe brain injury, besides the normalization of the intracranial pressure and maintenance of the blood pressure, it is necessary to prevent hyperventilation by administration of neuroplegic drugs and avoid anemia by means of blood transfusion.

\section{REFERENCIAS}

1. ANTHONY, A. J.; VENRATH, H. - Funktionsprüfungen der Atmung. J. Ambrosius Barth, Leipzig, 1962. 2. ASTRUP, P. - Klin. Wschr., 35:749, 1957. 3. BARTELS, H.; BUCHERL, E.; HERZT, C. W.; RODEWALD, G.; SCHWAB, M. - Lungenfunktionsprüfungen. Springer Verlag, Berlin-Göttingen-Heidelberg, 1959. 4. BERNSMEIER, A.; SIEMONS, K. - Klin. Wschr., 31:166, 1953. 5. BISCHOFF, W. - Zur Entstehung des "neurogen" ausgelösten akuten Lungenödems und der akuten MagenDarmblutungen. Tese de Docência, Köln, 1962. 6. BLAND, J. H. - Störungen des Wasser- und Elektrolythaushaltes. Georg Thieme Verlag, Stuttgart, 1959. 7. BROWN, 
H. W.; PLUM, F. - Amer. J. Med., 30:849, 1961. 8. COOK, A. W.; BROWDER, E. J.: LYONS, H. A. - J. Neurosurg., 18:366, 1961. 9. FROWEIN, R. A. - Hefte Unfallhk., 55:111, 1956. 10. FROWEIN, R. A. - Conscience et circulation dans les déplacements en masse du cerveau. IXe Réunion Europ. d'Informat. ElectroencéphaJegraph., Marseille, 12-10-1960. 11. FROWEIN, R. A. - Acta neurochir., 9:468, 1961. 12. FROWEIN, R. A. - Acta neurochir., Supl. 6, 1961. 13. FROWEIN, R. A. Klin. Med., 17:336, 1962. 14. FROWEIN, R. A. - Kreislaufstörungen bei akuten traumatischen Hirn-schädigungen. Symposium "Klinik und Therapie der Kollapszustände", Mainz 2-2-1962. 15. FROWEIN, R. A. - Zentrale Atemstörungen bei Schädel-Hirn-Verletzungen und bei Hirntumoren. Monograph. Ges. Geb. Neurol. u. Psychiatr., Vol. 101. Springer Verlag, Berlin-Göttingen-Heidelberg, 1962. 16. GÄNSHIRT, H. - Die Sauerstoffversorgung des Gehirns und ihre Störung bei der Liquordrucksteigerung und beim Hirnödem. Monograph. Ges. Geb. Neurol. u. Psychiatr., Vol. 81. Springer Verlag, Berlin-Göttingen-Heidelberg, 1957. 17. GÄNSHIRT, H.; TONNIS, W. - z. Nervenhk., 174:305, 1956. 18. GELIN, E. - Flüssigkeitsersatz im Schock. In v. Euler, U. S.; Bock, K. D. - Schock. Ciba Symposium, Stockholm, 1961. Springer Verlag, Berlin-Göttingen-Heidelberg, 1962. 19. HEYMAN, A.; BIRCHFIELD, R.; SIEKER, H. - Neurology 8:694, 1958. 20. HIRSCH, H.; GLEICHMANN, U.; KRISTeN, H.; MAgazinoviC, V. - Pflügers Arch. ges. Physiol., 273:213, 1961. 21. KETY, S. S.; SHENKIN, H. A.; SCHMIDT, C. F. - J. Clin. Invest., 27:493, 1948. 22. MAAS, A. H. J.; van HEIJST, A. N. P. - Clin. Chim. Acta 6:31-33 e 34-37, 1961. 23. NIELSEN, K. C. - Acta Med. Scand., 170:770, 1961. 24. NOELL, W.; SCHNEIDER, M. - Arch. Psychiat. u. Neurol., 180:713, 1948. 25. OPITZ, E.; SCHNEIDER, M. - Erg. Physiol., 46:126, 1950. 26. PAMPUS, F. - Veränderungen des Blutvolumens und der Blutzusammensetzung nach akuten schweren Kopfverletzungen. Beiträge z. Neurochir., 1:89, 1959. 27. PLUM, F.; SWAMSON, A. G. Arch. Neurol. a. Psychiat., 81:535, 1959. 28. SCHNEDORF, J. G.; MUNSLOW, R. A.; CRAWFORD, A. S.; McClURE, R. D. - Surg. Gyn. Obstet., 70:628, 1940. 29. SCHNEIDER, M. - Zur Pathophysiologie der verschiedenen Schockformen. Blut (em impressão). 30. SCHNEIDER, M. - Verh. Dtsch. Ges. Kreislauff., 19:1, 1953. 31. SCHNEIDER, M. - Acta neurochir., Supl. 7, 1960. 32. SCHURMANN, K. - Besondere Kreislaufreaktion im akuten Stadium der gedeckten Schädel-Hirnschädigungen. Neurochirurgenkongress Bad IschI, 3-9-1962. 33. SMOLIK, E. A.; MUETHER, R. O.; NASH, F. P.; KONNEKER, W. - Surg. Gyn. Obstet., 102:263, 1956.34. SMOLIK, E. A.; NASH, F. P. - J. Neurosurg., 17:669, 1960. 35. STRUGHOLD, H. - Klin. Wschr., 23:221, 1944. 36. TONNIS, W.; SCHIEFER, W. - Zirkulationsstörungen des Gehirns im Serieangiogramm. Springer Verlag, Berlin-Göttingen-Heidelberg, 1959. 37. WIGGERS, C. J. - Physiology of Shock. Cambridge, Mass., 1950. 